\title{
EL SEMICANTO, USO PUBLICITARIO DE UNA VENDEDORA AMBULANTE
}

\author{
Rodolfo Noriega Borja \\ Universidad Distrital Francisco José de Caldas \\ rodolfonobo@hotmail.com \\ Carlos Daniel Ortiz Caraballo \\ Corporación Universitaria Minuto de Dios \\ cd_ortiz@hotmail.com
}

\begin{abstract}
Resumen
El siguiente artículo es un estudio de caso que centra su análisis en los estribillos o pregones publicitarios de una vendedora ambulante en Bogotá, Colombia. Su objetivo es definir el semicanto como un fenómeno fonético - fonológico, a partir del análisis espectrográfico realizado a la muestra de voz extraída a la vendedora, con el fin establecer el modo en que se dispone el sonido en estos enunciados. Mediante la descripción de la duración, la intensidad y la línea entonativa de los fonemas, como la determinación de la altura formantita de la voz hablada y la voz cantada se describe el modo en que operan el "acento de frase" y la entonación en estos enunciados o estribillos publicitarios de carácter coloquial. El artículo plantea que según el propósito que el emisor tenga con su enunciado, éste puede modificarse, de lo que resulta un nuevo acento y entonación, o un semicanto.
\end{abstract}

PALABRAS CLAVES: acento, entonación, "acento de frase", voz cantada y voz hablada.

\begin{abstract}
This article is focused on the analysis of the advertising refrains of a woman, who works as a hawker in Bogota - Colombia. The objective of this paper is to define the semisinging as a phonetic - phonological phenomenon, based on the espectrographic analysis done to the voice sample recorded from the hawker, with the goal to establish the way sounds occured in these utterings. Throughout the description of the duration, intensity and pitch line of the phonemes, also the identification of the heigh core of the speech and the singing it is described the way "sentence stress" and entonation are presented in this kind of advertising messages or no formal advertisement. This article take into account that according to the speaker's speech goal, the speech can be modified which produces a new stress and entonation or what we call semisinging.
\end{abstract}

KEY WORDS: accent, intonation, "sentence stress", semisinging, spoken and sung voice.

"No se penetra enteramente en el dominio de una lengua mientras no se conoce la intimidad de su entonación".

TOMAS NAVARRO

\section{Introducción}

Los mensajes promocionales de los vendedores ambulantes son una variedad de enunciados que posee una riqueza acentual y entonativa única. Su 
ritmo melódico rompe con los parámetros comunes de la cadencia de la voz hablada (Jackson Menaldi, 1992) y se asemejan a los del canto, pese a que no cumplen a cabalidad con el mismo rendimiento vocal. Estos se encuentran en un punto intermedio entre el canto y el habla, y en ocasiones, entre el canto y el grito. Es por esta razón que este tipo especial de enunciados podrían ser definidos como semicantos, pues en ocasiones, su morfología fónica se parece a la de las letanías, debido a que los vendedores disponen la voz para componer un tipo de acento y entonación propia, con el fin de hacerse oír en la calle.

En lo que concierne a los estudio de la voz, la fonética y fonología se han dedicado a contrastar las distintas formas en que se despliega un enunciado (habla/canto, susurro/grito, impostación/regularidad, etcétera), por lo que hasta el momento no hay una definición exacta para este tipo de fenómeno fónico que hemos denominado semicanto. El análisis espectrográfico que aquí se propone permitirá establecer una primera definición de lo qué es, cómo se origina y por qué se produce este tipo emisiones. Consideramos que en las muestras extraídas a la vendedora ambulante, el semicanto se presenta a la par con el "acento de frase" y con la entonación; de hecho, es un subproducto que se origina por la concurrencia de ambos fenómenos tonales. Precisamente estos tres fenómenos son los que serán evidenciados en el análisis espectrográfico.

El objetivo de este artículo es definir el semicanto como un fenómeno fonetico - fonológico, a partir del análisis espectrográfico realizado a una muestra de voz de una vendedora ambulante de la ciudad de Bogotá, para determinar el modo en que se dispone el sonido en estos enunciados. Los términos: acento y entonación como suprasegmentos ${ }^{1}$ (Cantero Serena, 2002: 23), voz cantada y voz hablada (Jackson - Menaldi, 1992) son los ejes teóricos de este estudio de caso. Mediante el análisis de espectrogramas se describirá la forma en que se presentan estos fenómenos fónicos en los enunciados (mensajes publicitarios) de una vendedora ambulante o comerciante informal, los cuales serán utilizados para sustentar las ideas acerca del semicanto.

\section{El acento y la entonación como suprasegmentos}

Lo primero será definir los conceptos de acento y entonación, pues existen diferentes enfoques y perspectivas desde los que se puede asumir un análisis en el campo de las ciencias fónicas. Para ambos términos nos adherimos a las teorización de Francisco Cantero Serena (2002) que los define como rasgos suprasegmentales de los sonidos de la lengua. El gran aporte del lingüista, a

1 En este estudio se utiliza el término Suprasegmentales (terminología norteamericana) y no prosódico (terminología de Martinet y de la escuela fonológica). 
nuestro parecer, es que considera tanto el acento, en especial el "acento de frase", y la entonación como una curva melódica y rítmica en el tiempo del enunciado. Por supuesto, estas dos curvas pueden ser susceptibles a confusión si no se tiene claro el modo en que funciona cada una. En pos de dilucidar cualquier tipo de imprecisión o ambigüedad, presentaremos un elenco de citas traídas de fuentes autorizadas, desde las que también se puede percibir una breve evolución de estos dos conceptos.

Empezaremos diciendo que el acento y la entonación son fenómenos tonales ${ }^{2}$ que hacen posible la caracterización de los procesos de producción de la voz de una lengua, un hablante o un grupo social determinado, como también de la intención comunicativa y el estado de ánimo de quien habla. Según Cantero Serena (2002: 15) estos fenómenos se caracterizan como los de mayor dificultad en el análisis de las ciencias fónicas, debido a sus diversas funciones e incidencia directa en la significación de los enunciados.

Una definición sencilla del Acento es la de esfuerzo intensivo que afecta a una determinada sílaba en la palabra. El Diccionario de lingüística de Cardona (1991) lo define como: rasgo prosódico que realza una unidad lingüística superior al fonema (sílaba, morfema, palabra, sintagma, frase). En otros términos, el acento funciona como elemento de contraste entre otras unidades lingüísticas del mismo nivel (unidad acentuada y unidad no acentuada). Esta definición es la base para entender la conceptualización acerca del "acento de frase" que será expuesta más adelante.

En cuanto a la entonación, una explicación simplificada es la de línea musical producida por una sucesión de tonos con los que pronunciamos una secuencia de palabras. Quilis (1993), desde un punto de vista teórico, corrobora lo expuesto, cuando precisa que "la entonación es la función lingüísticamente significativa, socialmente representativa e individualmente expresiva de la frecuencia fundamental en el nivel de la oración" (Quilis, 1993: 410). Estas variaciones del fundamental (o del tono) son producidas por la vibración de los pliegues vocales, que son apreciables cuando el tono es ascendente, descendente, en suspensión, o también, cuando se presenta un ascenso de la intensidad ${ }^{4}$ de los sonidos.

2 Del tono de la voz.

3 La cursiva pertenece a la fuente original.

4 Cabe aclarar que la intensidad se genera en función de la energía o de la fuerza que ha sido puesta en vibración para la producción de un sonido cualquiera, dicha fuerza de articulación se mide en decibelios o decibeles (dB). Por ejemplo, todas las vocales tienen una intensidad diferente, intensidad que no es estable, pues según la lengua en la que se realice la producción fonética y fonológica de la articulación sonora, ésta también varía. La intensidad fonológica de las vocales en español es la siguiente: $a=25,18(\mathrm{~dB}), e=24,74(\mathrm{~dB}), o$ $=22,43(\mathrm{~dB}), i=21,92(\mathrm{~dB})$ y $u=20,58(\mathrm{~dB})$. El sonido vocálico abierto y central [a] tiene una mayor intensidad, debido que: 1) a igualdad de abertura del tracto vocal, la articulación 
Por otro lado, Canfield (1988: 8) precisa que la entonación es el tono musical que compone una frase. A partir de un estudio comparativo de la entonación del español en los dos continentes (Europa y América), el autor asegura que en el caso del español ibérico existe una clara distinción entre el ritmo de la aseveración y la interrogación, como también una marcada diferencia entre pregunta absoluta y una relativa, que no se da en el español americano. En síntesis, la importancia de la entonación en la caracterización de una lengua como el castellano, radica tanto en la matización de las palabras como la función lingüístico-expresiva del mismo. Función que se puede observar en las cadenas fónicas del discurso enunciado por la vendedora, como se expone más adelante.

En esa misma línea teórica está Cantero Serena (2002). Además de lo antes expuestos, este lingüista considera la entonación como un recurso comunicativo elemental de la lengua, que forma un sistema fonológico especial, el cual sirve para la organización de enunciados de un modo diferente, en diferentes lenguas. En sus propias palabras la entonación

es el fenómeno lingüístico que constituyen las variaciones de tono relevantes en el discurso oral $[\ldots]$ se trata del principal elemento cohesionador del habla, que además cumple distintas funciones lingüisticas y expresivas en la comunicación oral (p. 15).

A partir de este breve recorrido por los conceptos enunciados se puede afirmar que, el acento y la entonación son productos del tono o frecuencia del fundamental $\left(f_{o}\right)$, en otras palabras, son rasgos accesorios de los sonidos. Lo anterior se explica del siguiente modo: los fonemas de una lengua están caracterizados por una serie de cualidades físicas básicas conocidos como segmentos: la cantidad, el timbre, la intensidad y el tono hacen parte de esas propiedades acústicas esenciales, que le otorgan características tales como: sonoridad, vibración, etcétera. Estas cualidades físicas caracterizan a los fonemas en sí mismos, mas no a la cadena fónica (sucesiones de fonemas) o a los enunciados directamente. En cambio, a la cadena fónica se le superponen rasgos como el acento y la entonación; ambos elementos se consideran efectos asociados a las cualidades físicas mencionadas. Por ejemplo, una cadena fónica como Hábito, será una palabra distinta según se pronuncie con mayor intensidad los núcleos silábicos: lá/, líl, ló/, re-

posterior es más intensa, 2) el grado de cierre es inversamente proporcional al de intensidad, 3) a mayor cierre menor intensidad, 4) las vocales que son núcleo silábico presentan mayor intensidad y las que no, una menor. Por consiguiente, las sílabas átonas presentan mayor intensidad, por las consonantes, y en las tónicas esta intensidad proyecta un (1) dB menos. 5 Sin embargo, existen casos en los que el acento es el resultado de la intensidad y no del tono de los sonidos, es por ellos que muchas investigaciones fónicas no aseveran una exclusividad del tono como responsable del acento. 
presentados en las siguientes sucesiones de fonemas: [ábito] Hábito, [abíto] Habito y [abitó] Habitó. Ese esfuerzo intensivo que afecta a una determinada silaba en la cadena fónica y que tiene la función de determinar el significado de una palabra, es una cualidad extrínseca de los fonemas, que depende de la intensión significativa del hablante y no del sonido per se.

De igual modo, sucede con grupos fónicos más complejos, por ejemplo, en el enunciado "vinieron a cobrar". Este enunciado tendrá un valor diferente

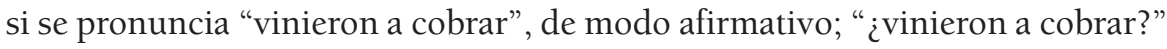
de modo interrogativo y "ivinieron a cobrar!" de modo exclamativo. En este caso, el ritmo melódico organiza cada enunciado de forma diferente y determina en cada uno de ellos su sentido. De igual modo, en el ejemplo, el efecto que se quiere lograr es el de significado o significación, pero, éste no es el único; la entonación también permite la elaboración de efectos estéticos, socioculturales, entre otros. En todo caso, a esos accesorios o efectos que se imprimen intencionalmente para complementar los sonidos se conocen como rasgos suprasegmentales.

\section{$2.1 \mathrm{El}$ "acento de frase"}

Dentro los suprasegmentos existen dos tipos distintos de acentos. El más conocido es el acento paradigmático o acento de la palabra, cuyo efecto en la cadena fónica fue ejemplificado anteriormente con los vocablos hábito, habito y habitó. El segundo caso es acento sintagmático o "acento de frase". Éste es un efecto tanto del acento de la cadena fónica como de la entonación. El "acento de frase" es el esfuerzo intensivo que afecta a todo un enunciado, es decir, que trasciende de los fonemas vocálicos hasta el grupo fónico ${ }^{6}$. En algunas ocasiones este tipo de acento puede ser confundido con la entonación o con una variedad entonativa sociocultural.

La forma en que el acento trasciende desde la palabra hasta el nivel del enunciado y sirve como puente de unión con la entonación es poniéndose de relieve sobre todos los acentos paradigmáticos que hay en una expresión determinada. Por ejemplo, en el grupo fónico anterior: "vinieron a cobrar" de modo interrogativo, el acento paradigmático de la primera cadena fónica está

6 Según Quilis (1993) establece una diferencia entre un grupo fónico y un grupo de entonación. Para él un "grupo fónico es la porción de discurso comprendida entre dos pausas" (Quilis, 1993: 418), mientras que un "grupo de entonación es la porción de discurso comprendida entre dos pausas, entre pausas e inflexiones del fundamental, entre inflexión del fundamental y pausa, o entre dos inflexiones del fundamental, que configura una unidad sintáctica más o menos larga o compleja (sintagma, cláusula, oración)" (Quilis, 1993: 418 - 419). Es decir, en un grupo de entonación puede contener a uno o varios grupos fónicos. 
sobre la primera vocal átona; es decir, sobre el sonido vocálico cerrado anterior /i/, y la segunda cadena fónica, sobre la primera vocal átona, o sea, en el fonema vocálico semicerrado posterior /o/. Sin embargo, el acento sintagmático de este enunciado recae sobre la última vocal atona /a/, que constituye una inflexión ascendente, como se ilustra en el esquema núm. 1, a continuación:

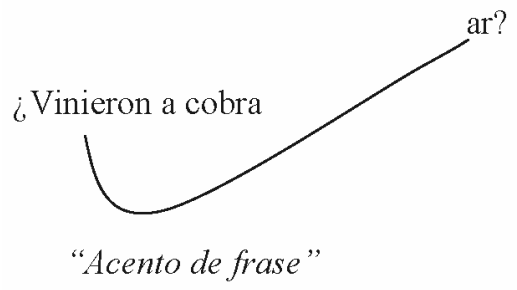

Esquema núm. 1. Grupo fónico con inflexión tonal interrogativa

A saber, a la segunda cadena fónica "cobrar" se le superpone el énfasis tonal del sintagma, lo cual produce un alargamiento o desdoblamiento de la vocal atona /a/, del ejemplo. Esto en algunos casos suele producir un ritmo o efecto distinto a los rasgos socioculturales, para establecerse como carácter estético, tal cual ocurre con los enunciados de la vendedora informal. Así, esta cadena fónica constituye el núcleo del enunciado, y a su vez, la palabra exacta que marca el ritmo del discurso. En ese sentido, el "acento de frase" se caracteriza por ser toda una inflexión tonal de una emisión de voz, mientras que el acento paradigmático es la inflexión de un fonema en una cadena fónica cualquiera. En el discurso conformado por dos o más grupos fónicos, el acento paradigmático es sólo el inicio de la inflexión tonal o "acento de frase".

En el caso del grupo fónico con inflexión tonal exclamativa: "ivinieron a cobrar!", el "acento de frase" recae sobre la vocal "a" atona, que está después del primer grupo fónico. Este fonema vocálico constituye el núcleo del enunciado, una inflexión ascendente que produce el desdoblamiento o descenso de éste, como se ilustra en seguida, en el esquema núm. 2:

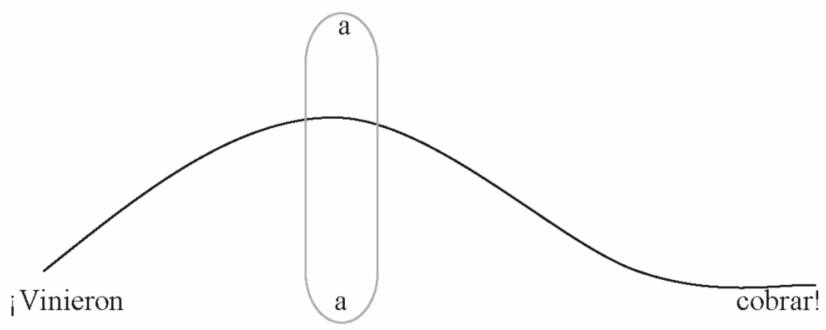

Esquema núm. 2. Grupo fónico con inflexión tonal exclamativa 
La razón para trabajar los rasgos suprasegmentales de esta manera conjunta en la muestra recogida, parte inicialmente del diagnóstico realizado a la misma, el cual sustentamos con Cantero Serena (2002), quien asegura que el acento y la entonación son fenómenos que se complementan. Según sus palabras, estos fenómenos fónicos tienen una jerarquía fónica definida, que funciona de la siguiente forma:

... el acento y la entonación funcionan solidariamente en lo que hemos llamado la función prelingüística de ambos fenómenos: el discurso, cuya forma material es la cadena hablada, no está constituido por una cadena de sonidos, dispuestos indistintamente uno tras otro, sino que tales sonidos, como hemos visto, están organizados jerárquicamente y forman bloques fónicos a tres niveles: en torno a las vocales, las sílabas; en torno a las vocales tónicas, las palabras fónicas; en torno a las inflexiones tonales, los grupos fónicos. Mediante estos bloques fónicos se estructura fónicamente el discurso, estructura que posibilita su comprensión por parte del oyente. Así, el primer contacto del oyente con el discurso es la estructuración entonativa, rítmica y acentual, ofrecida como una sucesión de bloques fónicos que sirven de contenedores a la estructura léxico-gramatical del discurso (p. 212 - 213).

En síntesis, el acento y la entonación ornamentan al discurso, con el único fin de hacerlo significativamente comprensible o estéticamente agradable y atractivo ${ }^{7}$. Tales marcas son de carácter dinámico, melódico, cuantitativo, silábico e influyen a todos los componentes fónicos de un acto de habla (Van Dijk, 2005) cualquiera, desde las unidades lingüísticas mínimas como el fonema, hasta las unidades mayores como la sílaba, la palabra, la oración, etcétera. Así, retomando nuevamente a Cantero Serena (2002: 23), el acento paradigmático afecta a la sílaba, el acento sintagmático al enunciado y la entonación, a toda la oración.

\section{Metodología}

Para el estudio de la muestra de voz se ha empleado el enfoque de análisis de la entonación por niveles $(\mathrm{AN})^{8}$, pues centra el análisis en el nivel tonal, la

$7 \quad$ Es el caso de la poesía, en el que los ritmos del verso se encuentran ordenados en concordancia al número de sílabas, a su acentuación y rima. El canto en un sentido general, está organizado en virtud de un ritmo fundado en la acentuación y la división de la frase; aunque para algunos géneros en especial son importantes también la notación por neumas y la tonalidad diatónica pura.

8 El modelo de Análisis por Niveles es una propuesta de Trager y Smith (1951), que se ha sido retomada por Quilis (1991). Según Navarro (2006) modelo de análisis por niveles (AN): el modelo de análisis por niveles hunde sus raíces en la tradición estructuralista norteamericana de base bloomfieldiana. En efecto, Bloomfield estableció una primera distinción entre sonidos y modificaciones, en la idea de que los sonidos articulados son sus- 
juntura y el acento 9 . Para ello se propone los siguientes pasos: 1) determinar el nivel específico de lengua en que se quiere observar. 2) establecer oposiciones de carácter semántico en los componentes del nivel de lengua seleccionado, con el fin de que el análisis de los elementos contrastado permita fijar o confirmar los resultados de la hipótesis.

En ese orden de ideas, después de seleccionar el fenómeno, se procedió a comparar la descripción de los niveles regulares de la voz hablada y la voz cantada que establece Jackson - Menaldi (1992) con los datos de duración, intensidad del nivel tonal y juntura que se obtuvieron de las muestras tomadas a la vendedora ambulante Luego se determina el comportamiento del "acento de frase" y la entonación, con el fin de corroborar en ellas la presencia del semicanto. Estos datos, como los de línea tonal fueron extraídos de la lectura de los espectrogramas.

\section{Análisis espectrográfico}

Antes de continuar se ha considerado pertinente hacer una breve descripción de los espectrogramas, para su total compresión. Las muestras de voz fueron sometidas al programa WASP, mediante el cual se hizo la transformación a espectrogramas. Básicamente, los espectrogramas que elabora este programa se encuentran compuestos por cuatro espectros o secciones, estas son:

1. Un espectro de amplitud de onda, en el cual se mide el tiempo (parte superior).

2. Un segundo espectro o espectro de banda ancha, en el que se encuentra la evidencia formántica, cuya medida va hasta los $51 \mathrm{db}$. (Parte media superior de la gráfica).

ceptibles de experimentar cambios vinculados "al espacio de tiempo a través del cual se continúa un sonido; la fuerza don la que se produce; el tono musical de la voz durante su producción" (bloomfield s 7.1). con lo que se establecía claramente la diferenciación entre segmentos y suprasegmentos.

9 Los tres niveles tonales propuestos por el modelo clásico son asumibles, entendiendo que os niveles 1 y 2 se aplican a entonaciones neutras y el nivel 3 se considera apara elementos enfáticos. En cuanto a los niveles tonales, el AN distinguen cuatro niveles tonales, que van desde el nivel 1 (el más grave) al nivel 4 (el más agudo); tales niveles no tienen un valor absoluto sino relativo. También son fonemas tonales las junturas (junctures) y el acento (stress, son cuatro niveles: primario, secundario, terciario y débil). En lo que se refiere a la junturas terminales van asociadas a un retardamiento en el tempo elocutivo de la última sílaba anterior a la pausa. El punto de anclaje de los niveles tonales corresponde a la sílaba más prominente, a partir de la cual se asignan dichos niveles otras posiciones, si bien la teoría debería implementarse con la consideración de curvas reales (nivel fonético que sirvan para establecer los patrones entonativos. Pag. 56-57. 
3. Un tercero espectro o espectro de banda estrecha, en la que se muestran los armónicos de cada formante, cuya medida va hasta los 56db., (Parte media inferior).

4. Y un último espectro o sección base, en la que se aprecia la línea tonal, cuya medida sobrepasa los $400 \mathrm{~Hz}$.

El filtro de banda ancha permite la descomposición de la onda de voz en armónicos, y el de banda estrecha, muestra la concentración de energía; en otras palabras, los formantes y sus respectivos núcleos. Valga anotar que la frecuencia fundamental $\left(\mathrm{f}_{\mathrm{o}}\right)$ es el principal patrón entonativo y se determina por sí misma o por sus múltiplos.

A continuación se describe el fenómeno del semicanto que se presenta en la muestra de voz tomada a la informante (vendedora informal). Valga aclarar que, la descripción debe partir del análisis del acento, el "acento de frase" y la entonación, pues como se dijo al principio, este fenómeno no ha sido analizado previamente.

\subsection{Los datos etnográficos del informante}

La muestra fue obtenida de una mujer de 55 años de edad de la ciudad de Bogotá, con un nivel educativo de séptimo grado de bachillerato y de ocupación vendedora ambulante de minutos a celular o voceadora de minutos, como se les conoce a los que se dedican a esta actividad. La caracterización básica de la muestra sonora de esta mujer, es un pregón que se ubica entre los límites de la voz cantada y la voz hablada. Jackson - Menaldi (1992) afirma que la diferencia entre la voz hablada y la cantada estriba básicamente en que,

... la voz cantada es bien colocada cuando la energía acústica se concentra sobre una zona precisa de formantes lo que permite tener el máximo de resonancia ("teoría del focus"), por lo tanto, el rendimiento vocal es bien notorio. La diferencia de calidad se debe a que la voz cantada necesita expandirse en una extensión de dos octavas y la voz hablada sólo se mueve en un ámbito de una octava (p. 180).

Es por lo anterior, que se considera que las muestras que aquí se analizan no corresponden a un canto, tampoco a la de un grito, ni mucho menos a un enunciado conversacional, pues éstas se expanden en una extensión de octava y media. Lo que significa que auditivamente estos pregones tienen características de todas las anteriores, o sea, de canto, habla y, por momentos hasta de grito, aunque en menor escala.

Los anuncios publicitarios que emite la vendedora ambulante se caracterizan por la utilización de frases cortas o apocopadas, con inflexión afirmativa - incitativa, con las que se anuncian los productos. Las muestras recogidas para el análisis espectrográfico son las siguientes: 1) "bolsas para la basura", 2) "llamadas a celular, llamadas" y 3) "llamadas a celular". 


\subsection{Análisis espectrográfico del acento}

En el primer bloque fónico obtenido "bolsas para la basura", el acento se manifiesta de la siguiente manera: las tres sílabas acentuadas que son: [b . ] - [p á.] - [ s ú.] se observa el contraste entre las unidades lingüísticas acentuables y las no acentuables. La primera cadena fónica lleva el acento en el fonema vocálico semicerrado posterior /o/, u sonido [o] atona, la segunda en fonema vocálico abierto central /a/, o "a" atona y la tercera, en el fonema vocálico cerrado posterior $/ \mathrm{u} /$, o sonido $[\mathrm{u}]$ atona. Estos fonemas vocálicos conforman los núcleos silábicos de estas cadenas.

En cuanto a la observación del acento en los espectrogramas, este rasgo se evidencia por la duración que presentan los núcleos silábicos de cada cadena fónica. Para ejemplificar: la duración del alófono del fonema vocálico semicerrado posterior /o/, u sonido [o], primer núcleo de la primera cadena [b . $\mathrm{s}$ a], es de 230,4 ms; mientras que el fonema vocálico abierto central /a/ es de $138,24 \mathrm{~ms}$, teniendo en cuenta que la informante hace una elisión del fonema fricativo alveolar sordo /s/. Aunque estas vocales obedecen a fonemas diferentes, podrían manejar rasgos acústicos muy similares por sus características de acentuación y sobre todo si se tiene en cuenta su duración.

$\mathrm{El}$ acento en la siguiente cadena fónica presenta un contraste con mayor precisión, ya que son dos vocales medias, es decir, el fonema vocálico abierto central /a/ es enunciado con una menor abertura. Por esta razón, en la segunda cadena fónica [p á. a], el núcleo silábico tiene una duración de 172,8ms, mientras que el fonema vocálico siguiente, sólo alcanza los 15,2ms. Es así como se ve en el espectro de banda angosta del espectrograma núm. 1:

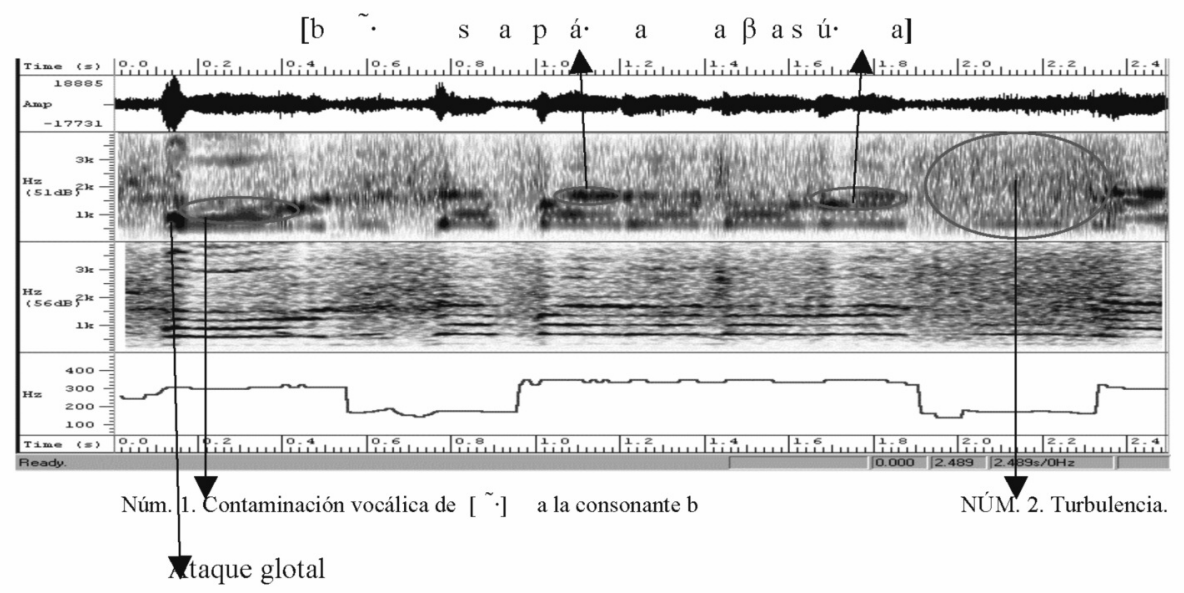

Espectrograma núm. 1. Bolsas para la basura 
Según el espectro de voz anterior, el inicio del discurso de la vendedora se ejecuta de manera hablada. A medida que se articula, se establece la elevación de la fuerza e intensidad de la producción del sonido que origina la formación del núcleo formántico, el cual logra alcanzar hercios más elevado que en el discurso hablado (actos de habla), pero en una escala menor de hercios, en relación a la ubicación de los formantes en el canto. Justamente esta ubicación intermedia conlleva al fenómeno que evidencia espectrográficamente, lo que en este estudio de caso ha sido denominado un semicanto. La acentuación de los sonidos vocálicos [ . ] contamina los sonidos posteriores, de manera tal, que los núcleos tienden a convertirse en una sola franja plana y ennegrecida (fenómeno núm. 1, en el espectrograma). La última parte del espectrograma no evidencia claramente núcleos formánticos, debido a efectos turbulentos de la articulación de sonido (fenómeno núm. 2, en el espectrograma), pero se retoma la formación de núcleos en la última vocal en la que la turbulencia desaparece, lo que permite apreciar una mayor duración del fonema, al tiempo que la presencia del "acento de frase" que recae sobre él.

En cuanto a la segunda muestra recogida: "llamadas a celular, llamadas", presenta semejanza en la primera y la tercera cadena fónica, o sea, en llamadas [ ã $\mathrm{m} \cdot \mathrm{a}$ ]. Con los otros núcleos silábicos correspondientes a este enunciado se evidencia el mismo fenómeno de la duración del acento, a través de la duración las sílabas tónicas. Al igual que la muestra anterior, en ambas cadenas se presenta la elisión del fonema fricativo alveolar sordo /s/. Los resultados que arrojó el espectro de banda angosta del espectrograma núm. 2, permitió corroborar este fenómeno.

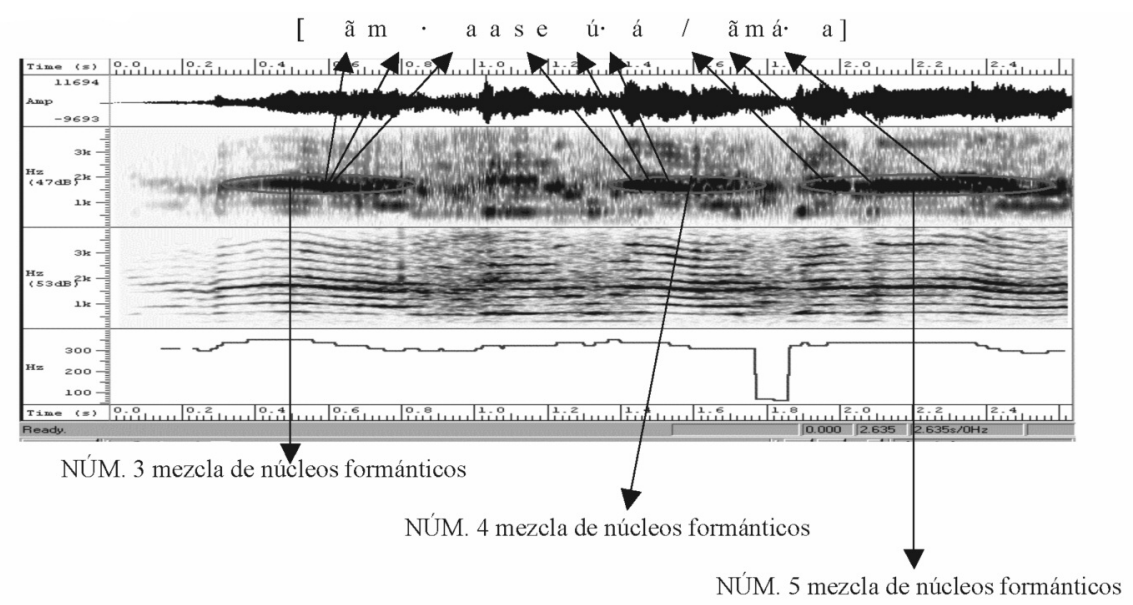

Espectrograma núm. 2. Llamada a celular, llamadas 
Como se puede apreciar, en el espectrograma no es del todo sencillo establecer con precisión la extensión de cada núcleo formántico. Efecto que conduce a determinar un sólo núcleo en el espectro (fenómeno núm. 3, 4 y 5, en el espectrograma). Esto sucede en una misma altura de hercios, lo que evidencia, una vez más, la ejecución de un semicanto y no un fenómeno de voz hablada o voz cantada. Por tanto, el "acento de frase", que es determinado por la sílaba de mayor fuerza de intensidad en la articulación, no resulta fácil de establecer, por la homogeneidad que supone el ennegrecimiento de todos los núcleos. En el primer espectro, la definición de formantes es más clara, ya que le semicanto se ejecuta de manera un tanto pausada.

Además, en esta segunda muestra "llamadas a celular, llamadas" [ ã m . a a s e ú. á / ã má. a ], se encontró que la informante hace uso del acento enfático o de insistencia, en la primera y en la última cadenas fónicas. Los tres núcleos silábicos que conforman el primer morfema [ ã $\mathrm{m} \cdot \mathrm{a}$ ] evidencian una duración diferente, esto es debido a la doble acentuación. Por ejemplo, la primera acentuación es en la sílaba [m ·], (número 4, en el espectrograma núm. 2), que arrojó una duración de $127,05 \mathrm{~ms}$, y la segunda acentuación es en la sílaba [ a], cuya duración es de $287,98 \mathrm{~ms}$. En el segundo morfema de este grupo fónico [ s e ú. á ], el fonema vocálico cerrado posterior [ú] (número 5, en el espectrograma núm. 2), es la segunda sílaba tónica y tiene una duración de 135,52ms. En ésta última se nota claramente que en contraste con la segunda acentuación [á] tiende a ser mayor el alargamiento del núcleo silábico, esto ocurre debido a la intencionalidad que pretende el hablante. Además, cabe tener en cuenta, que la informante hace elisión del fonema lateral palatal sonoro / /, lo que refuerza aún más la segunda acentuación y el alargamiento del núcleo silábico final. Alargamientos que produce el semicanto, a partir del "acento de frase" y la entonación que hace la hablante.

En cuanto a la tercera muestra recogida "llamadas a celular" [ ã $\mathrm{m} \cdot \mathrm{a} a$ s e ú. á ], los resultados resultan ser similares a los del segundo grupo fónico "llamadas a celular, llamadas", en su primer morfema. En síntesis, se presenta la elisión del fonema fricativo alveolar sordo /s/, el núcleo silábico de la sílaba ma [m ·] (número 6, en el espectrograma núm. 3) tiene una duración de $127,05 \mathrm{~ms}$, menor a la del núcleo silábico de la sílaba da [ a] que es de 287,98ms, debido a la doble acentuación del morfema; también se presenta el uso del acento enfático, en ambas cadenas fónicas. Asimismo, el segundo morfema de este grupo fónico [ s e ú. á ] se mantiene con dos núcleos silábicos acentuados y con los mismos valores en la duración acentual que en el grupo fónico analizado arriba. Esto es, para la segunda sílaba tónica [ú], una duración de 135,52ms., y un segundo núcleo silábico acentual [á] de 165,72ms., con la 
característica de la elisión del fonema lateral palatal sonoro / /. Es así como se evidencia en el espectro de banda ancha y banda estrecha del espectrograma núm. 3 , en el que la intensidad acentual se muestra con un mayor ennegrecimiento de los formantes.

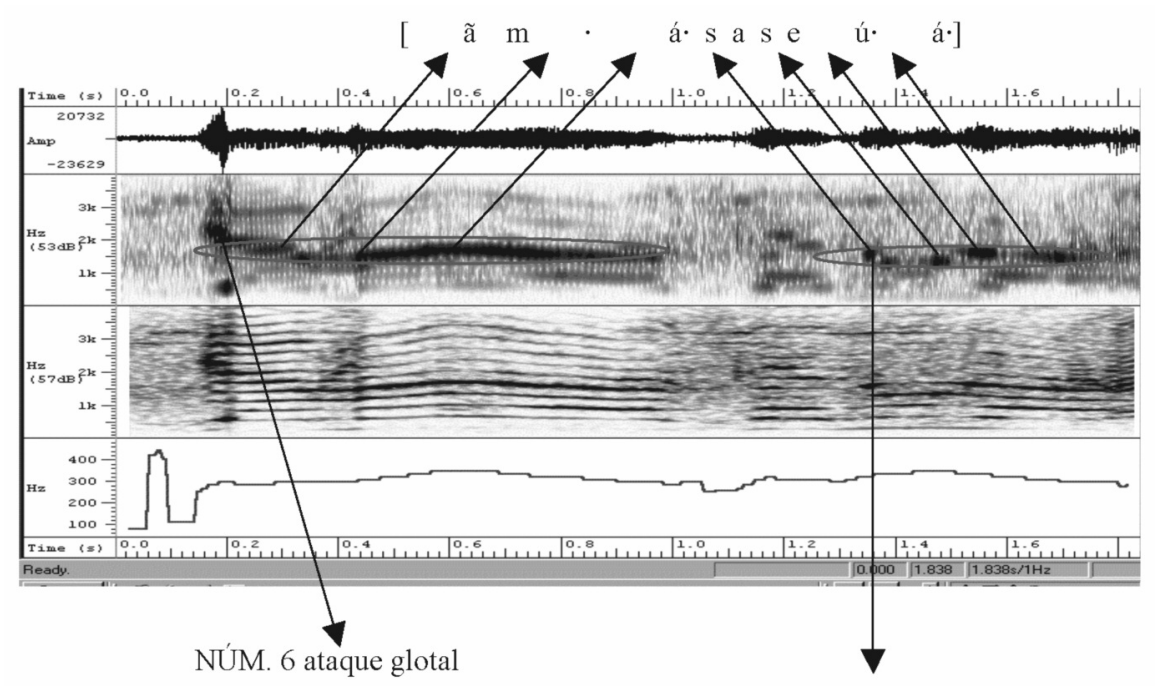

NúM. 7 ataque glotal

Espectrograma núm. 3. Llamadas a celular

Al igual que el espectrograma anterior, la determinación formántica se caracteriza por ser un sólo y extenso núcleo, con lo que se evidencia el semicanto. Al inicio de cada fonema (sonogramas de frases núm. 1 y 3 ) se presenta una posición formántica diferente, que podríamos llamarlo un mini-núcleo o mayor concentración de energía, el cual es producido por un ataque glotal, el cual es identificado con un punto de mayor ennegrecimiento (fenómeno núm. 6 y 7 , en el espectrograma). Los espectrogramas lo presentan al inicio de cada fonema y aún más, cuando es un fonema sonoro y acentuado o núcleo formántico. Esto también confirma la presencia del semicanto, pues si bien podría ser un formante extra (correspondiente a la voz cantada), éste está por debajo de los formantes de esta categoría. En los espectrogramas uno y tres se pueden apreciar este formante, en posiciones diferentes al núcleo general.

Con el análisis anterior se corrobora lo mencionado por Quilis (1993: 388), cuando teoriza sobre el acento y propone la duración como un elemento constitutivo de este rasgo suprasegmental. Claro está que el acento, aunque no es el principal constituyente de la silaba, es tan valioso como la intensidad, 
ambos elementos le sirven de soporte a la frecuencia del fundamental $\left(\mathrm{F}_{\mathrm{o}}\right)$, como quedará demostrado en el análisis que viene a continuación.

\subsection{Análisis espectrográfico de la intensidad de la onda, entonación}

Si se observa con detenimiento el reflejo de la intensidad de la onda en las muestras espectrográficas, se nota que la intensidad está en función de la energía o de la fuerza que se ha puesto en vibración (fuerza de articulación), que es medida en decibeles o decibelios (db). Por ejemplo, en el espectro de "Ilamadas a celular, llamadas" se puede comparar la primera vocal tónica en la sílaba [m ·], con la vocal átona de la silaba [ á.]. De este modo es posible determinar que la primera sílaba es la de mayor intensidad, pues ella está en los $21,15 \mathrm{db}$ y la segunda, en unos $13 \mathrm{db}$. En esa muestra espectrográfica, la informante hace una doble articulación, puesto que utiliza el acento de intensidad, los núcleos silábicos de cada morfema así lo corroboran. Obsérvese las vocales tónicas de llamadas [m · á.], las cuales presentan la misma intensidad 19,87db. Esta intensidad de la onda se puede apreciar en el espectro de amplitud de onda de los espectrogramas núm. 4 y núm. 5 :

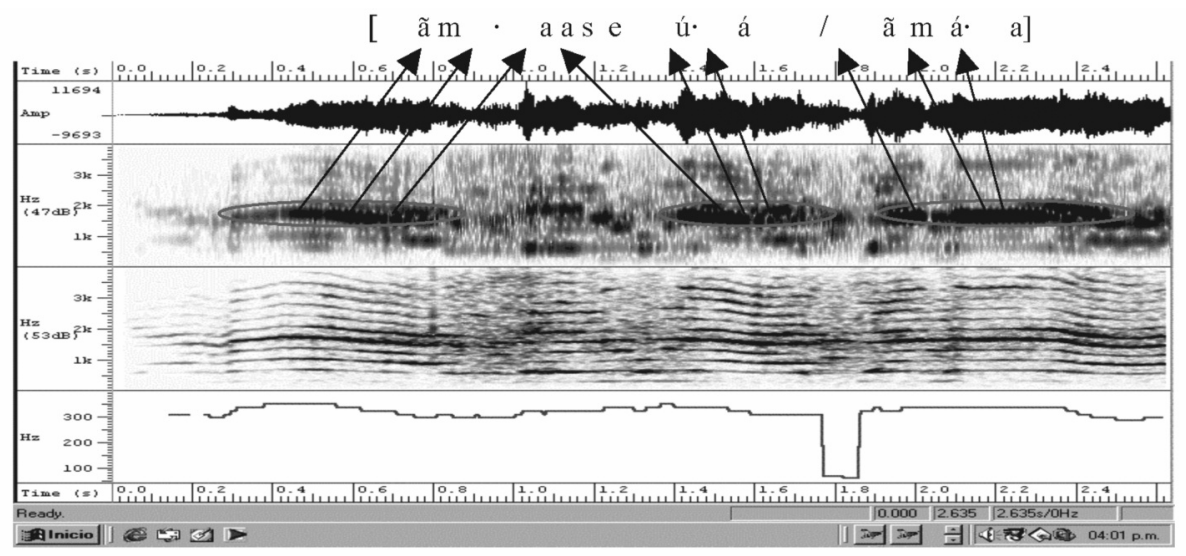

Espectrograma núm. 4. Llamadas a celular, llamadas

Según el espectrograma anterior, en el enunciado de la vendedora no se logra establecer la intensidad por sílabas, como se podría hacer en un espectrograma de voz hablada, ya que se presenta un único formante extenso, con una misma intensidad en toda la frase. Además, éste carece o no presenta el formante extra que debería llevar una muestra de voz cantada, ya que no es un canto como tal. 


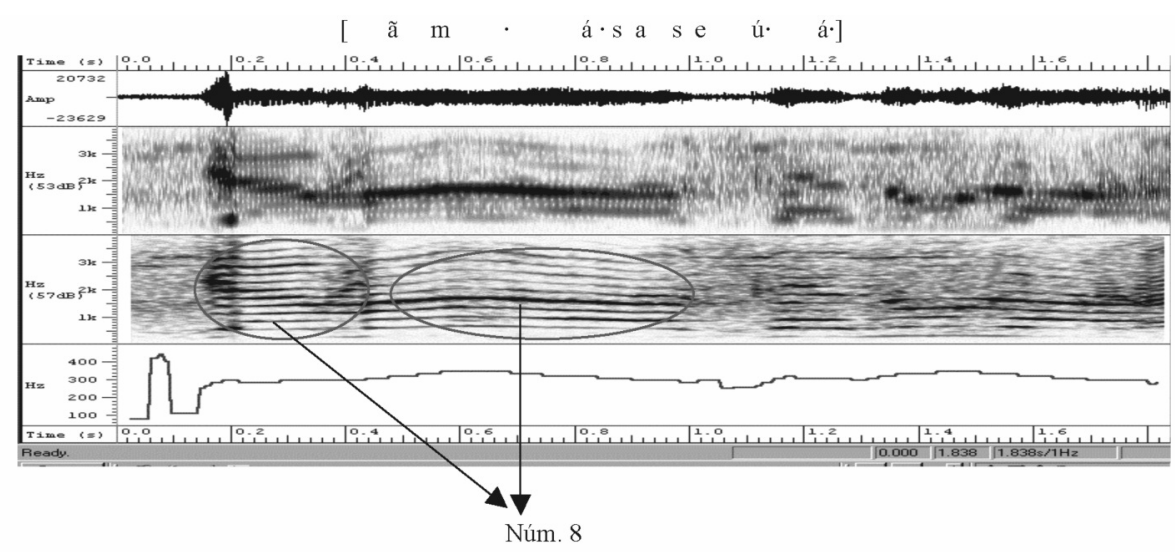

Espectrograma núm. 5. Llamadas a celular

El vibrato (Jackson Menaldi, 1992: 170) es la modulación de baja frecuencia de la voz, exactamente, el control que hace el cantante de ciertos sonidos articulados, los cuales se caracterizan porque en un 1/4 de tono tiene de 7 a 8 vibraciones por segundo, $\mathrm{y}$ de dos a tres decibelios de intensidad. Pese a la presencia del vibrato en el espectrograma anterior, éste no corresponde a los de un cantante, pues su número de vibraciones es menor, lo cual implica una desafinación o descontrol de los llamados gallos, en todo caso un sonido muy desagradable, que es percibido fácilmente. Dado que el formante presenta un número mayor, de 10 a 11 vibraciones por segundo, se evidencia que no es un canto como tal; de hecho, calificarlo como el vibrato de un cantante, sería un desatino, pero como es semicanto (fenómeno NÚM. 8, en el espectrograma), implica que tiene ciertas cadenas fónicas rítmicas y otras arrítmicas.

\subsection{Análisis espectrográfico de la entonación y "acento de frase"}

¿Con qué fin los vendedores anuncian sus productos de esta forma? La vendedora ambulante vocea, es decir, enuncia con intención apelativa, pero recuérdese que su enunciado no va dirigido a alguien en particular, sino a todos las personas que transitan a su alrededor. Por lo tanto, la línea tonal que se registra en dicho enunciado, pertenece a una entonación intencional o sea, hecha para llamar la atención de los otros. Lo que demuestra que la entonación es creada de modo estratégico, es decir, con un fin específico que sobrepasa el carácter regional o social establecido en la entonación de la voz hablada, para establecerse en lo estético, en una especie de impostación de la entonación y el "acento de frase", que si bien supera las características del discurso hablado, no alcanza los del canto. 
Lo anterior se evidencia en la muestra de voz "bolsas para la basura" que constituye un sólo grupo fónico sin pausa. Éste presenta una línea tonal que inicia en suspensión, a unos $260 \mathrm{~Hz}$ y se mantiene por unos $564,4 \mathrm{~ms}$.; más adelante desciende hasta unos $180 \mathrm{~Hz}$, luego vuelve a mantenerse en unos 403,2ms., para ascender hasta unos $140 \mathrm{~Hz}$ y mantenerse en una suspensión irregular por unos 956,16ms. Por último, desciende y asciende otra vez. Esto prueba que el informante manejó un tono variado y sin pausa, en el mismo grupo fónico, realizando las junturas pertinentes. Como se muestra en la línea tonal núm. 1:

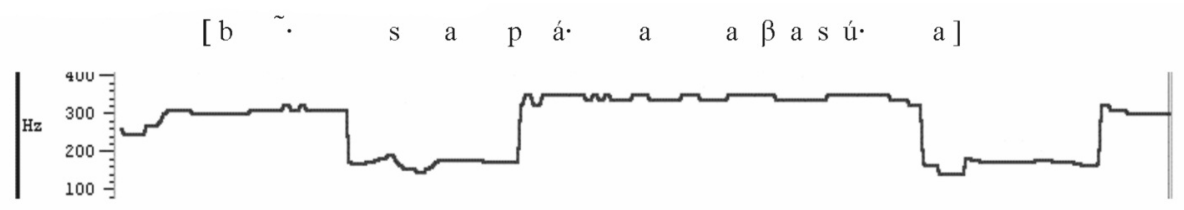

Espectrograma de la línea tonal núm. 1

Según la curva de la línea tonal, se puede asegurar que el acento de frase de este enunciado recae sobre la última sílaba. Esto hace que los acentos paradigmáticos que componen este grupo mantengan la sonoridad y el ritmo de este bloque fónico. Los picos del herciaje de la línea tonal así lo demuestran.

En el espectro de la línea tonal "llamadas a celular, llamadas" se caracteriza por ser un grupo fónico con pausas, cuya línea tonal se inicia a unos $310 \mathrm{~Hz}$, asciende a $360 \mathrm{~Hz}$, desciende a $320 \mathrm{~Hz}$ y se mantiene hasta la pausa, (descanso del hablante) y retoma el mismo herciaje, hasta el final del grupo fónico. Así se evidencia en la línea tonal núm. 2:

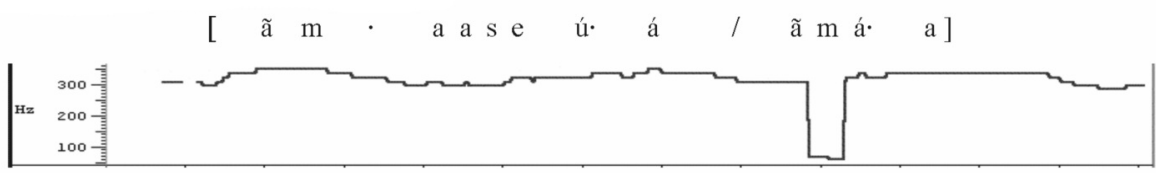

Espectrograma de la línea tonal núm. 2

De acuerdo con la línea tonal, este enunciado es más establece. Esto hace que su "acento de frase" y su línea entonativa sean una sola; en otra palabras, que no haya muchas variantes más allá de la pausa respiratoria del hablante (descenso precipitado en la línea tonal). De este modo, la base de sonoridad rítmica es la repetición de palabras, en este caso en particular, se hace referencia a la repetición de la palabra "llamada".

Por último, la muestra "llamadas a celular" es un grupo fónico sin pausas y con junturas apropiadas. La línea tonal inicia a $80 \mathrm{~Hz}$, asciende bruscamente 
hasta los $480 \mathrm{~Hz}$ y desciende hasta $100 \mathrm{~Hz}$. Luego vuelve a ascender a $310 \mathrm{~Hz}$, manteniéndose de forma irregular hasta el final de este grupo fónico. En el espectrograma de la línea tonal núm. 3 se percibe lo explicado:

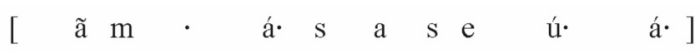

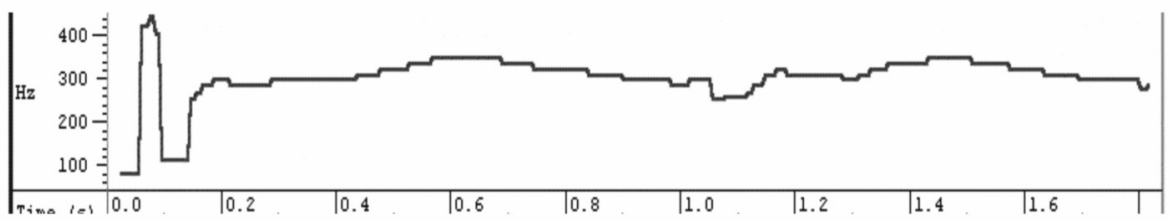

Espectrograma de la línea tonal núm. 3

Según esta línea tonal, el acento de frase se superpone a la entonación cuando recae sobre la última sílaba del enunciado, que se percibe en el último pico de la línea tonal (núm. 3), con su respectiva prolongación. Esto demuestra que la intención del hablante se inclina hacia la producción de enunciados rítmicos o estéticamente sonoros que sobrepasen la elaboración de un enunciado netamente hablado, pero que no lleguen al grito. Esto demuestra la conciencia del hablante con la entonación de su enunciado; él sabe que si grita aunque sea un poco, ahuyente a los potenciales clientes.

\section{Resultado preliminar del análisis espectrográfico}

En la lectura de espectrogramas se registró un rango de frecuencia que se elevó hasta los $4000 \mathrm{cps}$ (ciclos por segundo), en sus espectros de banda ancha y banda angosta o estrecha. A manera general, se puede afirmar que existe en la muestra un manejo plano y homogéneo del tono en la entonación intencional. Con el espectro de los armónicos de cada muestra, se aprecia que es una voz grave, que no suena como una voz muy femenina o de niño, esto se sustenta en que cuanto más grueso es el espectro del armónico, más grave la voz, y cuanto más delgados son los espectros de los armónicos más aguda es la voz. En el espectrograma número cinco se puede notar en la descomposición armónica espectrografía el grosor de dichos armónicos, lo cual indica una voz grave. Por otra parte, la intensidad se muestra con un mayor ennegrecimiento de los formantes. A causa del tono y la acentuación hay una curva melódica homogénea, como también, algunos alargamientos en los núcleos vocálicos.

La mayor concentración de energía en una determinada zona formántica es propia de la voz cantada, como también un desplazamiento de los formantes hacia un mayor nivel en hercios, lo que manifiesta con la revelación de un formante 
extra, entre los formantes núm. $3\left(\mathrm{f}_{3}\right)$ y el núm. $4\left(\mathrm{f}_{4}\right)$. Lo anterior se corrobora con la afirmación de Jackson-Menaldi (1992), cuando asegura que,

... los formantes de la voz cantada generalmente están desplazados en relación a los de la voz hablada, su dirección es variable. Agrega, basada en Sundberg, que el primer formante se encuentra entre los $250-700 \mathrm{~Hz}$; el segundo formante se encuentra entre los $700-2.500 \mathrm{~Hz}$; el extra pico fue designado "siging formant", una inserción de un extra formante ubicado entre el tercero y cuarto (p. 180).

En este caso, la vendedora no presenta el "singing formant" porque no está cantando, lo cual prueba que su enunciación es tan sólo un desplazamiento del formante núm. $1\left(\mathrm{f}_{1}\right)$, en otras palabras, el hablante no está cantando, pero tampoco está hablando. En las tres muestras recogidas, el herciaje de los formantes núm. 1 empieza a marcar desde los $5000 \mathrm{~Hz}$ en adelante. Otro rasgo característico de la enunciación del informante es la prolongación de las vocales, característica propia del canto, a diferencia del habla, en la que se resaltan las consonantes sin la mencionada prolongación.

Los núcleos de los formantes en la voz cantada se ubican en lugares diferentes en el espectrograma, además son de mayor y diferente herciaje, pues están ubicados entre los $250 \mathrm{~Hz}$ y $700 \mathrm{~Hz}$ para el primer formante, y los $700 \mathrm{~Hz}$ a $2500 \mathrm{~Hz}$ para el segundo, aún más para el tercero. Según los estudios en el área, en una conversación, la altura de las vocales de los hombres oscila entre los $110 \mathrm{~Hz}$ y $164 \mathrm{~Hz}, 220 \mathrm{~Hz}$ y $330 \mathrm{~Hz}$ para la mujer. La formación de los núcleos formánticos en los 5 espectrogramas de este trabajo no está en el rango antes mencionado. Adicionalmente, los formantes no están en orden aleatorio o en posiciones diferentes como sucede en la voz cantada y en la voz hablada, en la muestra obtenida, estos se encuentran a la misma altura, bastante unidos, lo que hace difícil establecer dónde inicia y dónde termina cada uno.

De la voz cantada el informante presenta entonación, acentuación y algo de modulación precisa. La ubicación de los formantes núm. 1 hasta el núm. 4 es muy reducida en todas las vocales, algo diferente de lo que puede ocurrir en la voz hablada, en la que cada formante tiende a guardar su respectiva distancia entre los otros (Jackson Menaldi, 1992). Por lo tanto, el informante no está ubicado en el nivel acústico de un cantante, tampoco en el de un hablante, sino en un intermedio (semicanto), debido al pregón o estribillo discursivo usado que va de acuerdo a su intensión discursiva.

Esta forma de enunciar de la vendedora es lo que se podría definir como la suma y mezcla de todos los acentos paradigmáticos con la entonación, en un "acento de frase". Al parecer, para su actividad lucrativa se necesitara un tipo especial de acentuación y entonación. Pues, el "acento de frase" que se refleja 
a través de la línea de entonación, tuvo una acentuada tendencia a ser plano, homogéneo, pero muy marcado como se apreció en las imágenes antes expuestas (número 7, en el espectrograma núm. 5). Con ello se modificó o rompió la entonación habitual de los hablantes de la ciudad de Bogotá.

En general, se puede decir que la vendedora ambulante tiene un manejo empírico de la entonación y el acento de frase, que utiliza en pos de su beneficio comercial. Pues existe en la muestra un uso deliberado del acento enfático o de insistencia como refuerzo de ciertos grupos, por ejemplo, "llamadas" [ ã m . a], aspecto que le da un realce especial a dicho enunciado, lo que hace que el producto quede expuesto abierta y contundentemente, según la forma del comercio o venta informal.

\section{Semicantos}

A partir del análisis anterior es posible afirmar que el semicanto es la modificación del acento de frase y la entonación, causado por la intención voluntaria del hablante y con algún fin específico, vender en este caso. Se puede aclarar que esta alteración que hace la vendedora ambulante no coincide con la entonación regional habitual de habla bogotana.

El semicanto se presenta a la par con el "acento de frase" y con la entonación; de hecho, es un subproducto que se origina por la concurrencia de ambos fenómenos tonales. En otras palabras, es un enunciado que fluctúa entre lo hablado y lo cantado, casi letánico. Las características sobresalientes de este fenómeno tonal son: a) presenta particularidades tanto de la voz hablada como de la voz cantada, a nivel fónico, b) el espectro de voz revela una línea tonal en permanente suspensión que se encuentra entre el habla y el canto y c) Mayor duración en el uso de los acentos.

\section{Conclusiones}

Al inicio de este análisis se expuso que alguno de estos enunciados son rítmicos y melódicos, y pese a que no están hechos con los mismos fines estéticos de la poesía y el canto, tienen la misma función en el fondo, agradar o llamar la atención de un modo penetrante y original, que romper o sobresale con el bullicio de la calle. Enunciados muy similares a aquellas arengas como: "extra, extra" de los jóvenes voceadores de prensa o "Vendo Alegría con coco y anís, casera cómpreme, cómpreme a mí, que vivo en el barrio de Getsemaní" de las palenqueras de Cartagena de Indias, siendo que éstas últimas arengas son un poco más elaboradas estéticamente en el ritmo y la rima, que las que acabamos de analizar, quizás por eso resulten más llamativas para el oído de los clientes. 
A partir de la lectura de los espectrogramas se puede concluir que, esta vendedora ambulante reviste su expresión con el "acento de frase". En este caso, el uso del lenguaje busca emitir una información, exactamente, los nombres de los productos que se están promocionado (bolsas para la basura y minutos a celular). Así, la vendedora al mencionarlos se ubica en una comunicación unidireccional que al hacer uso de algunas funciones del lenguaje o demostrar su eficacia de su intención apelativa, pasa a ser bidireccional. De este modo, llama la atención de los receptores.

Pese al papel trascendente que adquiere la sílaba en la conformación de un ritmo, con un fin comunicativo particular, ésta no pierde el acento a voluntad de un artificio estilístico, contrariamente lo mantiene siempre, sin importar los propósitos del emisor. Es así que la modulación o impostación de la entonación de la voz se impone como la modalidad publicitaria por excelencia en los vendedores ambulantes, pues es mediante ese recurso que intentará captar la atención de los clientes potenciales, repitiendo sus anuncios de un modo que para ellos lo hace sonar atractivo a los oídos de los consumidores. La vendedora utiliza una de las técnicas elementales de la publicidad que es la repetición del mensaje, específicamente, los nombres de los productos en venta.

Como el propósito primordial de la vendedora, al modular la voz, es el de ser escuchada, no el de decorar la locución de sus productos, ella cree que está gritando, a la manera similar en que antiguamente se voceaban o promocionaban las noticas de los diarios, en las esquinas de la ciudad; por esta razón su fin secundario termina por ser el rasgo de mayor relevancia en este tipo de fenómenos fónicos, una mezcla de habla, canto, tonos altos, en fin, semicantos.

Para la vendedora ambulante la utilización de las frases cortas, con nombre de los productos, como lema le permite la promoción fácil de éstos, mediante una emisión rítmica que para ella no implica un mayor esfuerzo enunciativo. Esta forma de promocionar instaura un nuevo ritmo melódico que presenta una variedad en la extensión, la duración y una mayor elevación en la producción de un enunciado. Es por eso que su ritmo melódico rompe con los parámetros comunes de la cadencia del habla y se asemeja a aquellos enunciados elaborados con fines estéticos, poemas, letanías, etcétera.

\section{Referencias bibliográficas}

Canfield, Delos Lincoln (1988): El español de América: Fonética. Barcelona, Editorial Crítica.

Cantero Serena, Francisco José (2002): Teoría y análisis de la entonación. Barcelona, Publicaciones de la Universidad de Barcelona.

Cardona, G. R. (1991): Diccionarios de lingüística. Barcelona, Editorial Ariel. 
Crystal, D. (2000): Diccionario de Lingüística y fonética. Barcelona, Octaedro.

Gili Gaya, S. (1961): Elemento de Fonética General. Madrid, Gredos.

Jackson-Menaldi, M. C. A. (1992): La voz normal. Buenos Aires, Editorial Médica Panamerican.

Navarro, Antonio Hidalgo (2006): Aspectos de la entonación española: viejos y nuevos enfoques. Madrid, Arcos/libros.

NAVARRO TOMÁS, T. (1974): Manual de entonación española. Madrid, Ediciones Guadarrama.

Pilar Prieto (eds.) (2003): Teorías de la entonación. Barcelona, Editorial Ariel.

Quilis, A. (1993): Tratado de fonología y fonética española. Madrid, Gredos.

Ricardo-Torres, Otto (2004): El otro lado del habla: la entonación poética. Medellín, Fondo Editorial Universidad de Eafitg.

Van Dijk, A. Teun (1980): Estructuras y funciones del discurso. Barcelona, Siglo veintiuno editores. 